\title{
Children with diseases of the upper gastrointestinal tract are more likely to develop feeding problems
}

\author{
Katerina Sdravou ${ }^{a}$, Elpida Emmanouilidou-Fotoulaki ${ }^{a}$, Maria-Rafaela Mitakidou $^{a}$, Anastasia Printzab, \\ Athanasios Evangeliou ${ }^{a}$, Maria Fotoulaki ${ }^{a}$
}

School of Medicine, Aristotle University of Thessaloniki, General Hospital "Papageorgiou”; School of Health Sciences, Aristotle University of Thessaloniki, Thessaloniki, Greece

\begin{abstract}
Feeding problems may give rise to a number of adverse effects with organic, psychological, developmental and social impact. Specific medical conditions, especially diseases of the gastrointestinal system, are known to be associated with feeding problems. We searched databases, including MEDLINE and the Cochrane Library, for papers published in English from 1990 to March 2018. Keywords included "gastrointestinal diseases", "gastroesophageal reflux disease", "eosinophilic esophagitis", "food allergy", "children", "infants", and "feeding problems". We concluded that children with diseases of the upper gastrointestinal tract, especially those with gastroesophageal reflux disease and eosinophilic esophagitis, are more likely to develop feeding problems. However, further epidemiological studies are needed to determine the prevalence and the specific characteristics of feeding problems in children with certain upper gastrointestinal diseases, as well as the exact causal relationship between them.
\end{abstract}

Keywords Feeding problems, gastrointestinal diseases, gastroesophageal reflux disease, food allergy, eosinophilic esophagitis

Ann Gastroenterol 2019; 32 (2): 1-7

\section{Introduction}

Feeding is an intricate process that requires the biological regulation of appetite and satiety, the availability of food, the transferring of food into the mouth, the preparation of bolus in the mouth, swallowing, digestion, and evacuation. It takes place in a socio-cultural context by which it is significantly affected [1]. A large number of biological, psychological, sociocultural and environmental factors interact, jointly shaping and interfering in this process of development [2,3].

The elimination or deregulation of any of these factors entails a variety of problems that can occur in a wide range of activities

${ }^{a} 4^{\text {th }}$ Department of Pediatrics, School of Medicine, Aristotle University of Thessaloniki, General Hospital "Papageorgiou" (Katerina Sdravou, Elpida Emmanouilidou-Fotoulaki, Maria-Rafaela Mitakidou, Athanasios Evangeliou, Maria Fotoulaki); ${ }^{\mathrm{b}} 1^{\text {st }}$ Otolaryngology Department, Medical Department, School of Health Sciences, Aristotle University of Thessaloniki (Anastasia Printza), Thessaloniki, Greece

Correspondence to: Katerina Sdravou, SLT, MSc, SpeechLab, Adrianoupoleos 55, 55133, Thessaloniki, Greece,

e-mail: sdravouk@tcd.ie

Conflict of Interest: None

Received 9 September 2018; accepted 22 November 2018; published online 15 January 2019

DOI: https://doi.org/10.20524/aog.2019.0348 related to feeding [1]. The manifestation of feeding problems includes a diversity of symptoms: for example, complete or partial food refusal, food selectivity, dietary restriction, adherence to behavior that does not correspond to the stage of development, tube feeding dependence, reduced appetite, lack of self-sufficiency in feeding, and dysfunctional behaviors during meals $[4,5]$. These problems may be accompanied by swallowing disorders [6,7].

Feeding problems are associated with a number of negative effects at the organic, psychological, developmental, and social levels. Even when they do not affect nourishment and growth, they are capable of significantly degrading the quality of life of the child and the family and may result in a negative relationship between parents and children [8]. Although the majority of children present no difficulty in feeding and growing, feeding disorders in early childhood are frequent. Feeding problems are estimated to occur in $25-35 \%$ of children with normal growth, whereas in children with growth disorders this may reach as high as $85 \%$ [9]. However, feeding problems comprise a heterogeneous group and the lack of a clear definition should be taken into consideration when estimating their prevalence [10]. This makes it difficult to identify, prevent and detect feeding disorders early in order to treat them effectively. The widely accepted opinion is that organic, environmental and behavioral factors interact and result in feeding problems [11]. Certain clinical studies point out the fact that specific medical conditions, especially diseases of the gastrointestinal 
system, are linked with feeding problems [12-39].The aim of this review was to examine whether children with upper gastrointestinal diseases are more likely to present with feeding problems. This article provides a brief overview of the association between feeding problems and upper gastrointestinal diseases. Based on the findings of this review, gastroesophageal reflux disease (GERD) and eosinophilic esophagitis are the leading organic causes of feeding problems in children with typical development [15-23,35-39]. Food allergy and intestinal motility disorders are also associated with feeding problems, but have not been the subject of systematic controlled studies [18,26-34,36].

\section{Materials and methods}

We conducted a review of articles published on pediatric feeding disorders from 1990 to March 2018. Electronic databases were searched, including MEDLINE, EMBASE, Cochrane Library and Google Scholar. The literature review was performed using the following MeSH terms: "gastrointestinal diseases", "gastroesophageal reflux disease", "eosinophilic esophagitis", "food allergy", "children", "infants" and "feeding problems". Initially 650 studies were retrieved. Studies of feeding disorders due to other etiologies, such as diseases of the lower gastrointestinal tract, neurological disorders, prematurity, autism, sensory problems and environmental issues, were excluded. Only studies published in English were included. Finally, 20 studies of feeding disorders in children with diseases of the upper gastrointestinal tract were selected. A flow chart illustrating the study selection process is shown in Fig. 1. Table 1 summarizes the studies evaluated.

\section{Results}

\section{Feeding problems in children with GERD}

Clinical manifestations of GERD in children include recurrent regurgitation, vomiting, abdominal pain, heartburn, poor weight gain, and irritability. When GERD symptoms remain untreated they are associated with a decrease in quality of life, for both the children and their families, and may lead to complications, such as reflux esophagitis, failure to thrive, esophageal stricture and Barrett's esophagus [12,13]. GERD may also cause decreased laryngopharyngeal sensitivity, laryngitis, sinusitis, increased frequency of silent aspiration, pulmonary infections, bronchiectasis and apnea, as well as asthma [14]. Furthermore, GERD is the disease most commonly linked with feeding pain; as a result, the biological need for feeding is overcome by the desire to avoid the annoying stimulus. Aversion is more intense when connected with oropharyngeal dysphagia and aspiration incidents [13].

A clinical study of 201 children with feeding problems verified that $76 \%$ of them had a certain medical disorder, with the majority (41\%) being GERD patients [15]. In a

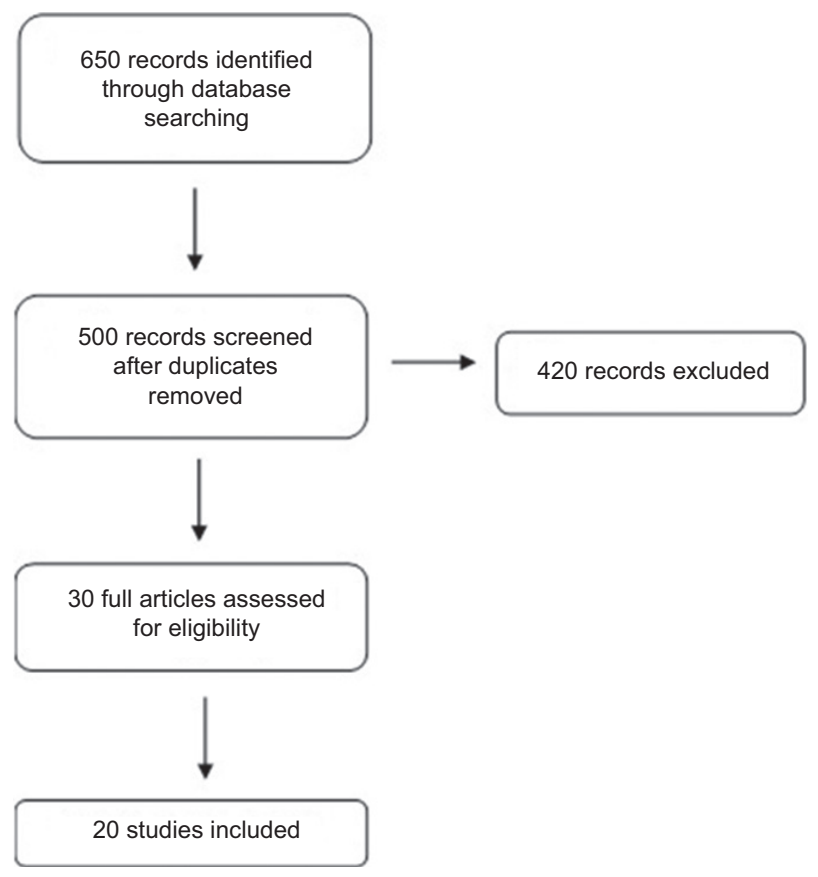

Figure 1 Study flow diagram

sample of 72 feeding tube-dependent children, organic causes were dominant: $83 \%$ of the sample had a diagnosis of oropharyngeal and gastrointestinal abnormalities and $64 \%$ had a diagnosis of cardiac, pulmonary, neurological or genetic disease [16]. A review of 38 intervention studies in the literature demonstrated that 212 of 218 participants with feeding problems who received treatment suffered a specific medical condition. Among these, 55\% were diagnosed with a gastrointestinal disorder, mainly GERD [17].Another study of childhood feeding problems demonstrated that the vast majority of the children (340 of 349) had a certain organic disorder and most commonly GERD (51\%). With regard to the type of feeding problem, $34 \%$ had food refusal, $21 \%$ selectivity by food type, $26 \%$ selectivity by food texture, $44 \%$ oral motor disorders, and $23 \%$ dysphagia. The frequency and severity of the feeding problem, and especially of food refusal, were greater in children with GERD than in those with other organic disorders. Specifically, $69 \%$ of the children who had food refusal also had GERD [18].

According to another study, children with GERD present with food refusal and selectivity, which persists after the treatment of the organic cause. Additionally, it is possible to develop oral and tactile hypersensitivity that aggravates the transition to solid food and hinders the acquisition of developmental feeding landmarks [19]. A study in infants with GERD pointed out that a number of participants developed food refusal, which resulted in faltering growth necessitating supplementary tube feeding [20]. In an observational study of feeding disorders in infants with regular regurgitations, it was ascertained that these infants were more likely to develop feeding disorders than were control subjects [21]. Another comparative study of 20 infants with GERD reported that their dietary intake was lower, not only than that of control 


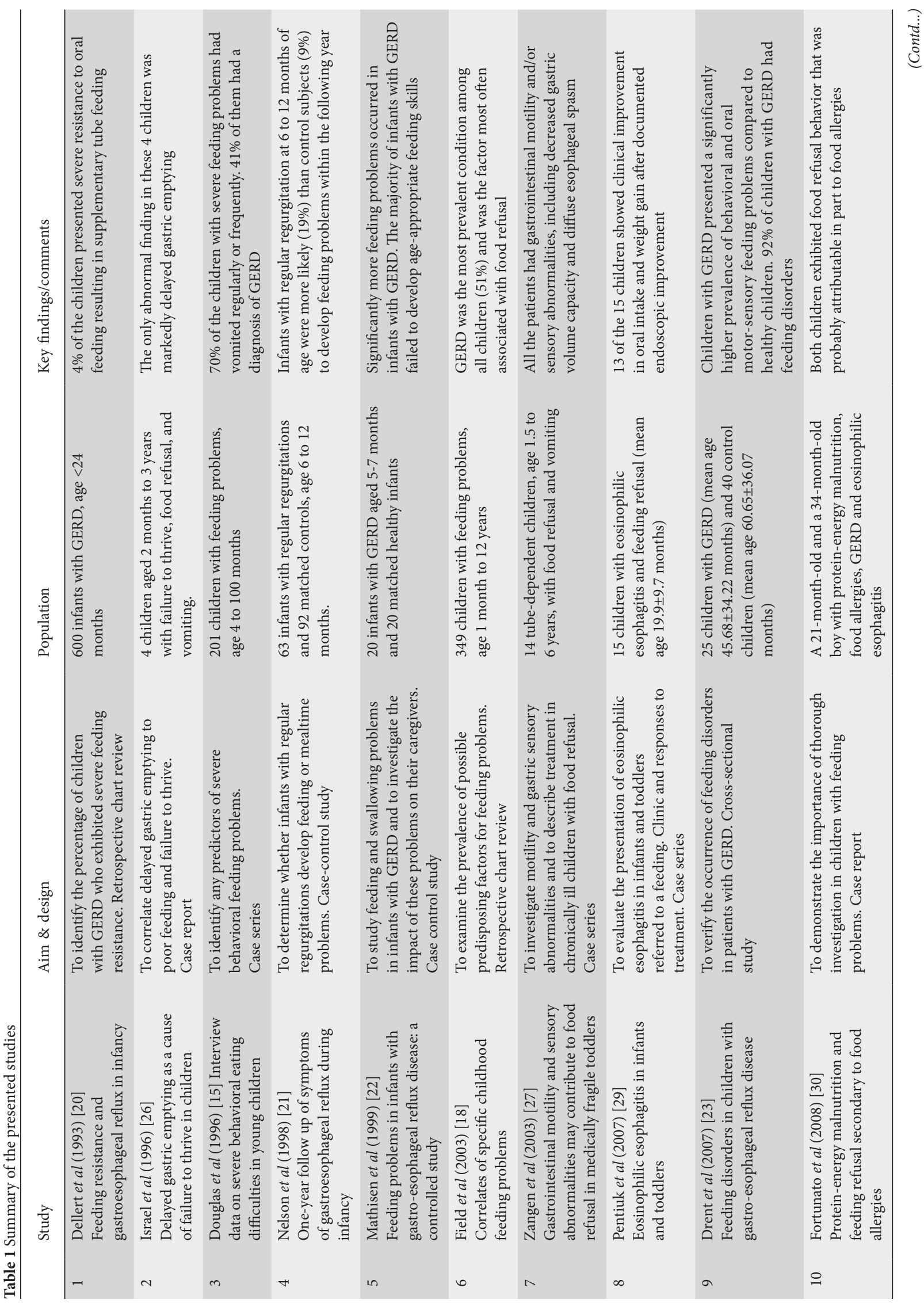




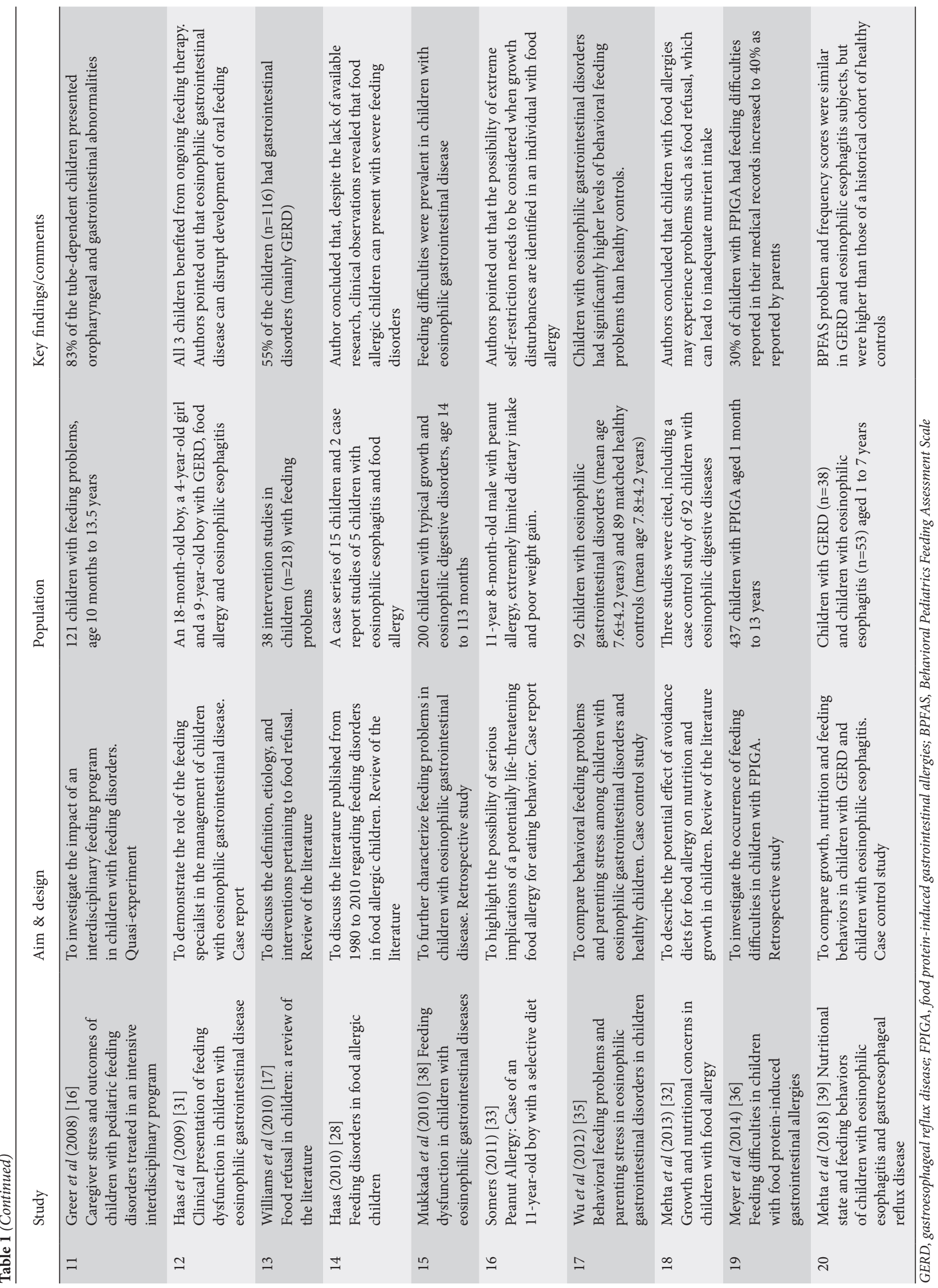


subjects, but also than the recommended dietary intake. Furthermore, the majority of infants with GERD had oral motor disorders, developed higher levels of food refusal, a greater number of choking incidents and interrupted meals. Delay in developmental stages, such as the ability to hold a spoon, putting objects into the mouth and feeding autonomy, was noticed as well. Moreover, their mothers reported negative feelings regarding the feeding process and found it necessary to be present during feeding. Videofluoroscopy findings identified mainly oral phase dysphagia, as well as silent aspiration and delayed esophageal transit [22]. Another observational study pointed out that children with GERD $(\mathrm{N}=25)$ were more likely to show behavioral feeding disorders and oral motor delays compared with the control group $(\mathrm{N}=40)$. Specifically, 92\% of children with GERD had feeding disorders, $44 \%$ of which involved behavioral issues and $81 \%$ oral motor problems. It is noteworthy that a high percentage of the control group (47\%) also had feeding problems: $40 \%$ behavioral and $10 \%$ oral motor deficits [23]. A possible explanation for this result might be the fact that the specific study aimed to identify a broad range of feeding problems, from mild to severe, and not necessarily feeding disorders of clinical significance.

\section{Feeding problems in children with intestinal motility disorders}

Intestinal motility disorders, such as decreased gastric motility and delayed gastric emptying, may not be clinically obvious but may lead to a decrease in appetite, early satiety and food refusal, according to some researchers [18,24-27]. Israel and Mahdi reported a case series of 4 children with noticeably delayed gastric emptying who presented with failure to thrive, food refusal and vomiting. All 4 children underwent a gastric drainage procedure, which resulted in a dramatic change in food intake and a decrease in vomiting [26].Field et al also reported 15 children with delayed gastric emptying who presented with feeding problems, including food refusal, selectivity and oral motor problems [18]. All of them also had GERD, which prevented the researchers from determining the impact of the delayed gastric emptying alone on the development of the feeding problems. The assumption of the authors was that the delayed gastric emptying might have exacerbated GERD and inhibited the appetite, or might have been the result of malnutrition in some of the children. Digestive tract motility disorders, including delayed gastric emptying, dysrhythmia of the antrum and diffuse esophageal spasm, were reported in 14 catheter-dependent children with food refusal who had episodes of vomiting and eructation even after fundoplication surgery [27].

\section{Feeding problems in children with food allergies}

According to the limited, but continually growing literature, children with food allergies may exhibit dysfunctional behavior, such as restricted diet, selectivity, feeding refusal, food neophobia and generally anxiety over feeding, which can lead to reduced dietary intake, affect growth and may persist after the withdrawal of allergenic food [18,28-34,36]. The first report on the issue came from a chart review study that found food allergy to be a cause of feeding difficulty. Specifically, food allergy was reported in $21 \%$ of children with feeding disorders and in $15 \%$ of children with feeding refusal. However, the study stated that children had food allergies without further discussing the methodology of diagnosis [18]. According to a review, only 3 published studies were found on this issue from 1980-2010 [28]. The first study involved 15 children with eosinophilic esophagitis, 7 of whom with food allergy, who exhibited symptoms such as feeding refusal, low weight gain, oral defense, and vomiting episodes [29]. The other 2 were case studies of 5 children with significant feeding disorders and undernourishment. All of them were diagnosed with multiple food allergies, GERD, and eosinophilic esophagitis [30-31]. Another review cited 3 studies suggesting that maladaptive feeding behaviors in children with food allergy might affect growth [32]. One was a case report of a patient with peanut allergy [33]. The second study addressed quality of life and anxiety in patients and families with peanut allergy [34], whereas the third study discussed feeding difficulties in children with eosinophilic digestive diseases, which are separate entities [35]. A retrospective study investigated the occurrence of feeding difficulties in 437 children with food protein-induced gastrointestinal allergies (FPIGA). The most common diagnoses included non-immunoglobulin E (IgE)mediated gastrointestinal food allergies and food proteininduced enterocolitis. The study indicated that $30 \%$ of children with FPIGA had feeding difficulties reported in their medical records, increasing to $40 \%$ reported by parents. The clinical manifestations of vomiting, constipation, rectal bleeding, and weight loss, the presence of extraintestinal manifestations and the number of foods eliminated from the diet were significantly associated with the presence of feeding difficulties [36].

\section{Feeding problems in children with eosinophilic esophagitis}

More recently, studies with strong research design provided high quality evidence to support the association between eosinophilic esophagitis and feeding problems. One casecontrol study investigated feeding disorders and parental stress in 92 children with eosinophilic digestive disorders using the Behavioral Pediatrics Feeding Assessment Scale (BPFAS) [35]. This scale is one of the most widely used and validated methods for detecting feeding problems [4,37]. According to the findings, children with eosinophilic disorders had significantly higher levels of behavioral feeding disorders compared to the control group. The presence of feeding disorders was more common in younger children and was positively associated with elevated levels of parental stress. A retrospective study in 200 children with typical growth and eosinophilic digestive disorders reported that $16.5 \%$ of children had severe feeding disorders. More specifically, about $94 \%$ exhibited dysfunctional behaviors such as food refusal, food selectivity and reduced 
dietary intake; $85 \%$ had a tendency to vomiting or vomiting episodes; while $21 \%$ were diagnosed with failure to thrive. Of these children, $88 \%$ had food allergy and 52\% other allergic diseases [38].

Another recent prospective study compared growth, nutrition and feeding behaviors in 91 children aged 1-7 with GERD and eosinophilic esophagitis using the BPFAS. Children with GERD and eosinophilic esophagitis had higher feeding behavior scores than healthy children and nearly one-third of the subjects had abnormal scores. Nevertheless, the GERD and eosinophilic esophagitis groups had both growth parameters within normal range and appropriate nutritional intake. BPFAS scores were not correlated with body mass index z-scores, age of presentation, or duration of the disease in either the GERD or the eosinophilic esophagitis group. However, a relatively strong correlation was found between weight-for-length z-score and the Parent Feelings/Strategies Frequency Score. Another interesting finding was that those on food allergen restriction diets reported significantly fewer problematic feeding behaviors than those on regular diets, suggesting that the type of eosinophilic esophagitis treatment had a significant effect on feeding behaviors. The problematic behaviors most frequently reported in both groups included taking longer than 20 min to finish a meal, preferring to drink rather than eat, and trying to negotiate what will be eaten. The child behaviors thought to be most problematic to parents included not enjoying eating, taking longer than $20 \mathrm{~min}$ to eat, and having a poor appetite. Most frequent problematic Parent Feelings/ Strategies observed were getting frustrated and anxious during mealtimes, coaxing their child to eat, and not feeling confident that their child was getting enough to eat. The parental feelings that were most problematic to parents were getting frustrated or anxious while feeding their child, not feeling confident that their child was getting enough to eat, and feeling that their child's eating patterns hurt his/her health [39].

\section{Concluding remarks}

This review focuses on feeding problems in children with upper gastrointestinal diseases and as such it raises an important issue in pediatric gastroenterology of clinical importance for pediatricians, speech and language therapists, nutritionists or dieticians, and all health professionals dealing with pediatric patients who have chronic gastrointestinal disorders or feeding difficulties. Several clinical studies reveal that children with gastrointestinal diseases, especially those with GERD and eosinophilic esophagitis, are more likely to present with feeding problems that have a negative impact on development, growth, and psychosocial dynamics. These studies suggest that all children with gastrointestinal diseases should be regarded as vulnerable, and highlight the importance of assessing and treating feeding problems among these children in order to prevent severe complications. This is especially important when we take into consideration that most parents of children with moderate-to-severe feeding problems do not seek professional help, probably because they consider feeding problems as a normal part of growing up [10]. A multidisciplinary approach is required to prevent the potential impact of feeding problems, improve growth, and reinforce successful reintroduction of food into the diet. Early detection and treatment of the gastrointestinal disease is crucial for the elimination of the associated feeding problems and their consequences. However, in a subset of children the associated symptoms persist, affecting feeding. In these cases an early referral to a feeding specialist is mandatory.

The existent literature provides poor quality evidence to establish a strong connection between food allergy and feeding difficulties. Food allergies might be associated with feeding difficulties, but a cause and effect relationship cannot be established robustly because of limitations such as weaknesses in study design, small sample sizes, sample variability and poor differentiation from eosinophilic esophagitis. Moreover, the diagnosis of food allergies is inadequately presented in many instances. Although the evidence of these studies is not sufficiently important to affect any clinical decision, it should function as an incentive to conduct further studies with larger samples and more robust study design.

A limitation of our review study is that we found only a limited number of case-control studies and limited information about the causal relationship between feeding disorders and gastrointestinal diseases, since most studies only reported organic factors affecting feeding, such as environmental and behavioral aspects. Moreover, we have focused on a limited range of gastrointestinal diseases. Therefore, the extent of feeding problems over the whole spectrum of gastrointestinal diseases has not been examined.

Future epidemiological studies should be carried out to determine the prevalence and specific characteristics of feeding problems in children with certain gastrointestinal disorders, such as GERD and food allergy. Further experimental studies are needed to clarify the exact causal relationship between feeding problems and gastrointestinal diseases. In addition, therapeutic options should be tested with a view to providing effective targeted interventions. Since current knowledge regarding the long-term consequences of feeding problems in this pediatric population is poor, experimental and followup studies should explore the long-term impact of feeding problems on growth and development in children with gastrointestinal diseases.

\section{References}

1. Rudolph CD, Link DT. Feeding disorders in infants and children. Pediatr Clin North Am 2002;49:97-112.

2. Berlin K, Davies W, Lobato D, Silverman A. A biopsychosocial model of normative and problematic pediatric feeding. Child Health Care 2009;38:263-282.

3. Bryant-Waugh R, Markham L, Kreipe RE, Walsh BT. Feeding and eating disorders in childhood. Int J Eat Disord 2010;43:98-111.

4. Crist W, Napier-Phillips A. Mealtime behaviors of young children: a comparison of normative and clinical data. J Dev Behav Pediatr 2001;22:279-286.

5. Mackner LM, McGrath AM, Stark LJ. Dietary recommendations 
to prevent and manage chronic pediatric health conditions: adherence, intervention, and future directions. J Dev Behav Pediatr 2001;22:130-143.

6. Arvedson JC. Assessment of pediatric dysphagia and feeding disorders: clinical and instrumental approaches. Dev Disabil Res Rev 2008;14:118-127.

7. Printza A, Kyrgidis A, Pavlidou E, Triaridis S, Constantinidis J. Reliability and validity of the Eating Assessment Tool-10 (Greek adaptation) in neurogenic and head and neck cancerrelated oropharyngeal dysphagia. Eur Arch Otorhinolaryngol 2018;275:1861-1868.

8. Equit $M$, Pälmke $M$, Becker $N$, Moritz AM, Becker S, von Gontard A. Eating problems in young children - a populationbased study. Acta Paediatr 2013;102:149-155.

9. Manikam R, Perman JA. Pediatric feeding disorders. J Clin Gastroenterol 2000;30:34-46.

10. Nieuwenhuis T, Verhagen EA, Bos AF, van Dijk MW. Children born preterm and full term have similar rates of feeding problems at three years of age. Acta Paediatr 2016;105:e452-e457.

11. Piazza C. Feeding disorders and behavior: what have we learned? Dev Disabil Res Rev 2008;14:174-181.

12. Malaty HM, O’Malley KJ, Abudayyeh S, Graham DY, Gilger MA. Multidimensional measure for gastroesophageal reflux disease (MM-GERD) symptoms in children: a population-based study. Acta Paediatr 2008;97:1292-1297.

13. Rosen R, Vandenplas Y, Singendonk M, et al. Pediatric Gastroesophageal Reflux Clinical Practice Guidelines: Joint Recommendations of the North American Society for Pediatric Gastroenterology, Hepatology, and Nutrition and the European Society for Pediatric Gastroenterology, Hepatology, and Nutrition. J Pediatr Gastroenterol Nutr 2018;66:516-554.

14. El-Serag HB, Gilger M, Kuebeler M, Rabeneck L. Extraesophageal associations of gastroesophageal reflux disease in children without neurologic defects. Gastroenterology 2001;121:1294-1299.

15. Douglas JE, Bryon M. Interview data on severe behavioural eating difficulties in young children. Arch Dis Child 1996;75:304-308.

16. Greer AJ, Gulotta CS, Masler EA, Laud RB. Caregiver stress and outcomes of children with pediatric feeding disorders treated in an intensive interdisciplinary program. J Pediatr Psychol 2008;33:612-620.

17. Williams KE, Field DG, Seiverling L. Food refusal in children: a review of the literature. Res Dev Disabil 2010;31:625-633.

18. Field D, Garland M, Williams K. Correlates of specific childhood feeding problems. J Paediatr Child Health 2003;39:299-304.

19. Strudwick S. Gastro-oesophageal reflux and feeding: the speech and language therapist's perspective. Int J Pediatr Otorhinolaryngol 2003;67(Suppl 1):S101-S102.

20. Dellert SF, Hyams JS, Treem WR, Geertsma MA. Feeding resistance and gastroesophageal reflux in infancy. J Pediatr Gastroenterol Nutr 1993;17:66-71.

21. Nelson SP, Chen EH, Syniar GM, Christoffel KK. One-year follow-up of symptoms of gastroesophageal reflux during infancy. Pediatric Practice Research Group. Pediatrics 1998;102:e67.
22. Mathisen B, Worrall L, Masel J, Wall C, Shepherd RW. Feeding problems in infants with gastro-oesophageal reflux disease: a controlled study. J Paediatr Child Health 1999;35:163-169.

23. Drent LV, Pinto EA. [Feeding disorders in children with gastroesophageal reflux disease]. Pro Fono 2007;19:59-66.

24. Saliakellis E, Fotoulaki M. Gastroparesis in children. Ann Gastroenterol 2013;26:204-211.

25. Waseem S, Islam S, Kahn G, Moshiree B, Talley NJ. Spectrum of gastroparesis in children. $J$ Pediatr Gastroenterol Nutr 2012;55:166-172.

26. Israel DM, Mahdi G. Delayed gastric emptying as a cause of failure to thrive in children. J Pediatr Gastroenterol Nutr 1996;22:115-116.

27. Zangen T, Ciarla C, Zangen S, et al. Gastrointestinal motility and sensory abnormalities may contribute to food refusal in medically fragile toddlers. J Pediatr Gastroenterol Nutr 2003;37:287-293.

28. Haas AM. Feeding disorders in food allergic children. Curr Allergy Asthma Rep 2010;10:258-264.

29. Pentiuk SP, Miller CK, Kaul A. Eosinophilic esophagitis in infants and toddlers. Dysphagia 2007;22:44-48.

30. Fortunato JE, Scheimann AO. Protein-energy malnutrition and feeding refusal secondary to food allergies. Clin Pediatr (Phila) 2008;47:496-499.

31. Haas AM, Maune NC. Clinical presentation of feeding dysfunction in children with eosinophilic gastrointestinal disease. Immunol Allergy Clin North Am 2009;29:65-75.

32. Mehta H, Groetch M, Wang J. Growth and nutritional concerns in children with food allergy. Curr Opin Allergy Clin Immunol 2013;13:275-279.

33. Somers LS. Peanut allergy: case of an 11-year-old boy with a selective diet. J Am Diet Assoc 2011;111:301-306.

34. King RM, Knibb RC, Hourihane JO. Impact of peanut allergy on quality of life, stress and anxiety in the family. Allergy 2009;64:461-468.

35. Wu YP, Franciosi JP, Rothenberg ME, Hommel KA. Behavioral feeding problems and parenting stress in eosinophilic gastrointestinal disorders in children. Pediatr Allergy Immunol 2012;23:730-735.

36. Meyer R, Rommel N, Van Oudenhove L, Fleming C, Dziubak R, Shah N. Feeding difficulties in children with food proteininduced gastrointestinal allergies. J Gastroenterol Hepatol 2014;29:1764-1769.

37. Sdravou K, Evangeliou A, Tentzidou K, Sotiriadou F, Stasinou E, Fotoulaki M. The behavioural paediatrics feeding assessment scale is a reliable and valid tool for Greek children. Acta Paediatr 2018;107:1653-1654.

38. Mukkada VA, Haas A, Maune NC, et al. Feeding dysfunction in children with eosinophilic gastrointestinal diseases. Pediatrics 2010;126:e672-e677.

39. Mehta P, Furuta GT, Brennan T, et al. Nutritional state and feeding behaviors of children with eosinophilic esophagitis and gastroesophageal reflux disease. J Pediatr Gastroenterol Nutr 2018;66:603-608. 